\title{
A FEW MORE PROPERTIES OF SUM AND INTEGRAL SUM GRAPHS
}

\author{
V. Vilfred ${ }^{1 *}$, A. Suryakala ${ }^{2}$, and K. Rubin Mary ${ }^{3}$ \\ ${ }^{1}$ St.Jude's College, Thoothoor - 629176 \\ Kanyakumari District, Tamil Nadu, India, \\ vilfredkamal@gmail.com \\ ${ }^{2}$ Sree Devi Kumari College for Women, \\ Kuzhithurai - 629 163,Tamil Nadu, India, \\ suryaps88@gmail.com \\ ${ }^{3}$ St.Judes College, Thoothoor - 629176 \\ Kanyakumari District, Tamil Nadu, India, \\ rubinjudes@yahoo.com
}

\begin{abstract}
The concepts of sum graph and integral sum graph were introduced by Harary. A sum graph is a graph whose vertices can be labeled with distinct positive integers so that the sum of the labels on each pair of adjacent vertices is the label of some other vertex. Integral sum graphs have the same definition except that the labels may be any integers. Harary gave examples of all orders of sum graphs $G_{n}$ and integral sum graphs $G_{-n, n}, n \in \mathbb{N}$. The family of integral sum graph was extended by Vilfred and in this paper we obtain a few properties of sum and integral sum graphs and two new families of integral sum graphs.

Key words: Supplementary vertices in a sum graph, integral sum labeling, triangular book with a book mark, fan graph with a handle.

Abstrak. Konsep graf penjumlahan dan graf penjumlahan integral diperkenalkan pertama kali oleh Harary. Sebuah graf penjumlahan adalah sebuah graf yang titiktitiknya dapat dilabelkan dengan bilangan bulat positif yang berbeda sehingga jumlahan dari label-labelnya pada tiap pasang titik-titik yang bertetangga adalah label dari suatu titik lain. Graf penjumlahan integral memiliki definisi yang sama kecuali dalam hal jumlahan dari label-labelnya adalah suatu bilangan bulat. Harary telah menunjukkan contoh-contoh dari semua orde dari graf penjumlahan $G_{n}$ dan graf penjumlahan integral $G_{-n, n}, n \in \mathbb{N}$. Keluarga graf penjumlahan integral telah diperluas oleh Vilfred dan dalam paper ini kami mendapatkan beberapa sifat dari graf-graf penjumlahan dan penjumlahan integral dan dua keluarga baru dari graf penjumlahan integral.
\end{abstract}

2000 Mathematics Subject Classification: 05C78, 05C75, 05C70. Received: 25-05-2013, revised: 01-06-2014, accepted: 14-08-2014. 
Kata kunci: Titik-titik pengganti, pelabelan graf penjumlahan, buku segitiga, graf kipas dengan pegangan.

\section{INTRODUCTION}

Harary [7],[8] introduced the concepts of sum and integral sum graphs. $A$ graph $G$ is a sum graph if the vertices of $G$ can be labeled with distinct positive integers so that $e=u v$ is an edge of $G$ if and only if the sum of the labels on vertices $u$ and $v$ is also a label in $G$. He extended the concept to allow any integers and called them as integral sum graphs. To distinguish between the two types, we call sum graphs that use only positive integers $\mathbb{N}$ - sum graphs and those with any integers $\mathbb{Z}-$ sum graphs (See [10]). For any non-empty set of integers $S$, we let $G^{+}(S)$ denote the integral sum graph on the set $S$ and $-G^{+}(S)=G^{+}(-S)$. For integers $r$ and $s$ with $r<s$ we also let $[r, s]$ denote the set of integers $\{r, r+1, \ldots, s\}$. Harary's examples of $\mathbb{N}$ - sum graphs are thus $G^{+}([1, n])=G_{n}$ and his $\mathbb{N}-$ sum graphs are $G^{+}([-r, r])=G_{-r, r}$ for $r \in \mathbb{N}$. (Note that his notation is modified and we write $G_{-r, r}$ for what he called $G_{r, r}$. See [10]). The extension of Harary graphs to all intervals of integers was introduced by Vilfred in [14]: for any integers $r$ and $s$ with $r<s$, let $G_{r, s}=G^{+}([r, s])$. We denote the sum graph $G^{+}([1, n])$ by $G_{n}^{+}$ when it is labeled and by $G_{n}$ when it is unlabeled.

In $G_{n}^{+}$, for $n \geq 2, d\left(u_{i}\right)=n-1-i$ if $1 \leq i \leq\left\lfloor\frac{n}{2}\right\rfloor$ and $d\left(u_{i}\right)=n-i$ if $\left\lfloor\frac{n}{2}\right\rfloor+1 \leq i \leq n$ where $\lfloor x\rfloor$ denotes the floor of $x$ and $u_{i}$ is the vertex with label $i, 1 \leq i \leq n$. Graphs $G_{n}^{+},\left(G_{n}^{+}\right)^{c}$ and $K_{n}=G_{n}^{+} \cup\left(G_{n}^{+}\right)^{c}$ for $n=3$ to 8 are given in Figures 1 to 6 . Different properties of sum and integral sum graphs are studied by several authors [2],[4],[7]-[23]. In [20], for $n \geq 4, G_{\Delta n}$ is introduced and defined as an integral sum graph of order $n$ having precisely two vertices each of degree $n-1 ; G_{-1,1}=K_{1}+\left(\left(-G_{1}\right)+G_{1}\right) \cong K_{3}$ without vertex labels is the only integral sum graph $G$ having more than two vertices, each of degree $|V(G)|-1$ [20]; it is proved that Fan, Dutch M-Windmill [18], Banana trees and union of stars [19] are integral sum graphs; in [14] maximal integral sum graph is studied; and number of cycles of length 3 [12] and of length 4 [21] in $G_{m, n}$ are obtained. For $m<0<n$ and $m, n \in \mathbb{Z}, G_{m, n}=K_{1}+\left(\left(-G_{-m}\right)+G_{n}\right)$ is an integral sum graph of order $(-m)+n+1$. Integral sum graphs $G_{0,7}, G_{-1,6}, G_{-2,5}$ and $G_{-3,4}$ are given in Figures 7 and 8. In this paper the underlying graph of an integral sum graph is obtained by removing all vertex labels. We obtain new properties of sum and integral sum graphs and two new families of integral sum graphs, triangular book of $n$ pages with book mark and fan graph with a handle. Now let us see the following definitions.

Two distinct vertices of sum graph $G^{+}(S)$ with $n$ as its maximum value of vertex labels are called supplementary vertices if the sum of their vertex labels = $n+1$. (See [10])

A graph $G$ is an anti-sum graph or anti $-\mathbb{N}-$ sum graph if the vertices of $G$ can be labeled with distinct positive integers so that $e=u v$ is an edge of $G$ if and only if the sum of the labels on vertices $u$ and $v$ is not a vertex label in $G$. An 
anti-integral sum graph or anti- $\mathbb{Z}-$ sum graph is also defined just as anti-sum graph, the difference being that the labels may be any distinct integers. Clearly, $f$ is an integral sum labeling of graph $G$ if and only if $f$ is an anti-integral sum labeling of $G^{c}$.

Two unlabeled graphs are said to be comparable if one is a subgraph of the other, while two labeled graphs are comparable if one is a subgraph of the other with the labels preserved. Clearly, any two Harary graphs $G_{m}^{+}$and $G_{n}^{+}$are comparable. In contrast, it is easy to check that labeled graphs $G_{3}^{+}$and $G_{2}^{+}+G^{+}(\{3\})$ are not comparable even though as unlabeled graphs $G_{3}$ is a (spanning) subgraph of $G_{2}+G^{+}(\{3\})$. We also observe that for all $r$ and $s$ with $0 \leq r \leq s, G_{r} \cup G^{+}([r+1, s])$ is a (spanning) subgraph of $G_{s}$.

$A$ graph $G$ is a split graph if its vertices can be partitioned into a clique and a stable set. A clique in a graph is a set of pair-wise adjacent vertices and an independent set or stable set in a graph is a set of pair-wise non-adjacent vertices [3]. $G_{n}$ and $G_{n}^{c}$ are split graphs. Clearly, [1,m], [1, $\left.m+1\right],[m+1,2 m],[m+2,2 m+1]$ are cliques and $[m+1,2 m],[m+2,2 m+1],[1, m],[1, m+1]$ are stable sets in $G_{2 m}, G_{2 m+1}, G_{2 m}^{c}, G_{2 m+1}^{c}$, respectively.

$A$ graph $H$ is decomposable into the subgraphs $H_{1}, H_{2}, \ldots, H_{n}$ of $H$, if no $H_{i}$ has isolated vertices and the edge set of $H$ can be partitioned into the subsets $E\left(H_{1}\right), E\left(H_{2}\right), \ldots, E\left(H_{n}\right), i=1,2, \ldots, n$. Graph $H$ is said to be $F$-decomposable, if $H_{i} \cong F$ for every $i, i=1,2, \ldots, n$. If $H$ is $\mathrm{F}$-decomposable, then we say that $F$ divides $H$ and we write $F / H[6]$.

When $k$ copies of $C_{n}$ share a common edge, it will form an $n$-gon book of $k$ pages, and it is denoted by $B(n, k)$ or $B_{n, k}$. The common edge is called the spine or base of the book. A triangular book $B(3, k)$ or $B_{3, k}$ consists of $k$ triangles with a common edge and can be described as $B(3, k)=S T(k)+K_{1}=P_{2}+\left(k \cdot K_{1}\right)$ where $S T(k)$ denotes the star with $k$ leaves. Triangular book $B(3, k)$ with the spine $(u, v)$ is denoted by $T B_{k}(u, v)=P_{2}(u, v)+\left(k . K_{1}\right)$. We call $T B_{0}=K_{2}$, a book without pages as the trivial book [11].

An $n$-gon book of $k$ pages $B(n, k)$ with a pendant edge terminating from a vertex of the spine is called an $n-$ gon book with a book mark. Triangular book $T B_{k}(u, v)$ with book mark $(u, w)$ is denoted by $T B_{k}(u, v)(u, w)$ or $T B_{k}^{*}(u, v)$ where $w$ is the pendant vertex adjacent to $u$. $T B_{k}^{*}(u, v)$ is of order $k+3$ and size $2 k+2$. $T B_{4}^{*}\left(u_{0}, v_{0}\right)$ is given in Figure 9 .

A fan graph $F_{n-1}$ is the graph obtained by taking $n-3$ concurrent chords at a vertex in a cycle $C_{n}, n \geq, 3$. The vertex at which all the $n-3$ chords are concurrent is called the apex vertex [23]. The fan graph $F_{n}=P_{n}+K_{1}$ and is an integral sum graph for $n \geq 2$ [18] where $P_{n}$ is a path on $n$ vertices. Integral sum labeling of $F_{5}$ is given in Figure 9 .

Fan graph $F_{n}$ with a pendant edge attached with the apex vertex is called $a$ fan with a handle or a palm fan and it is denoted by $F_{n}^{*}[11] . F_{5}^{*}$, fan graph $F_{5}$ with handle $u_{0} v_{0}$ is given in Figure 9. 
We consider simple graphs only. For all basic notation and definitions in graph theory, we follow [6] and for additional reading on related graph labeling problems, we refer to [5].

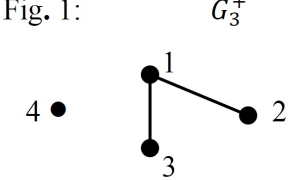

Fig. 2: $\quad G_{4}^{+}$

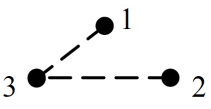

$\left(G_{3}^{+}\right)^{c}$

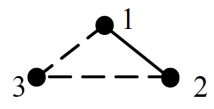

$\mathrm{K}_{3}=G_{3}^{+} \cup\left(G_{3}^{+}\right)$

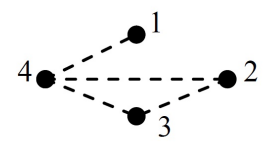

$\left(G_{4}^{+}\right)^{c}$

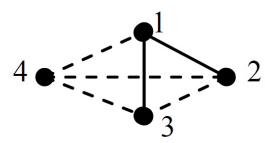

$\mathrm{K}_{4}=G_{4}^{+} \cup\left(G_{4}^{+}\right)^{c}$

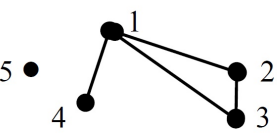

Fig. 3:

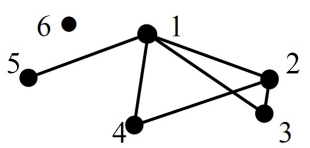

Fig. 4:

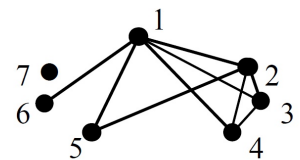

Fig. 5:

$G_{7}^{+}$

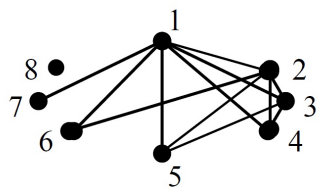

Fig. 6: $\quad G_{8}^{+}$

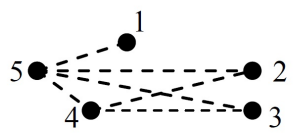

$\left(G_{5}^{+}\right)^{c}$

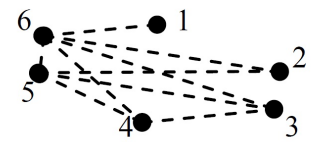

$\left(G_{6}^{+}\right)^{c}$

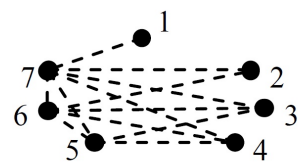

$\left(G_{7}^{+}\right)^{c}$

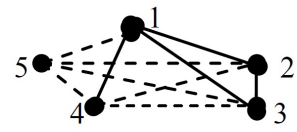

$\mathrm{K}_{5}=G_{5}^{+} \cup\left(G_{5}^{+}\right)^{c}$
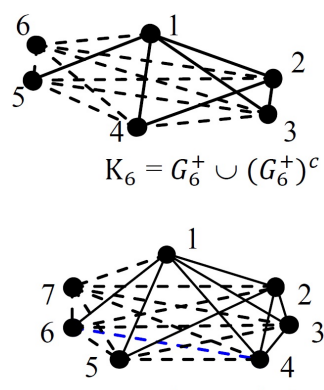

$\mathrm{K}_{7}=G_{7}^{+} \cup\left(G_{7}^{+}\right)^{C}$

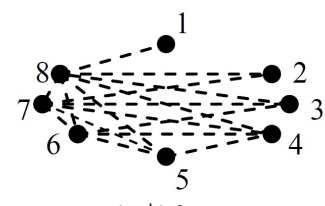

$\left(G_{8}^{+}\right)^{c}$

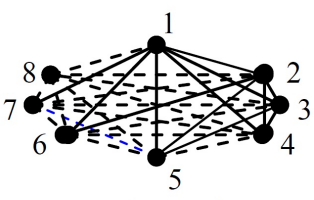

$\mathrm{K}_{8}=G_{8}^{+} \cup\left(G_{8}^{+}\right)^{c}$ 
Fig. 7:

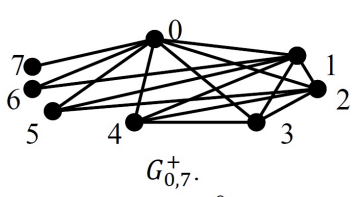

$G_{0,7}^{+}$

Fig. 8:
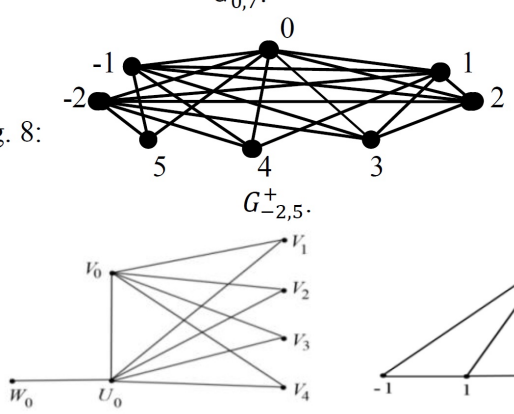

Fig. 9: $\quad T B_{4}^{*}\left(\mathrm{u}_{0}, \mathrm{v}_{0}\right)$.

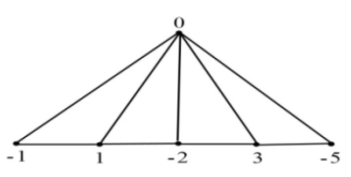

$F_{5}$.

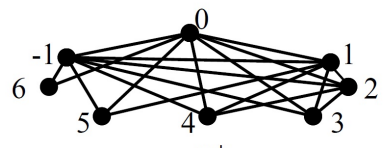

$G_{-1,6}^{+}$.
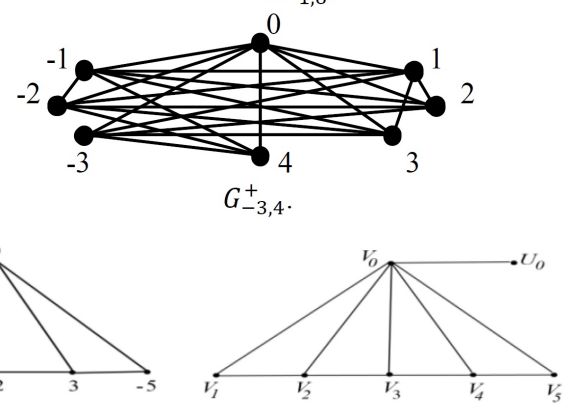

$F_{5}^{*}$.

\section{A Few Properties of Sum and Integral Sum Graphs}

We now turn to some subgraph relationships between graphs. For convenience, if graph $F$ is a subgraph of graph $G$ without the vertex labels, this will often be denoted by $F \subseteq_{w v l} G$. Two graphs are called non-comparable if neither is a subgraph of the other. If $F$ is an induced subgraph of $G$, this will sometimes be denoted by $F \leq G$ and by $F \leq{ }_{w v l} G$. Similarly, if $F$ is congruent to $G$ without the vertex labels, we often write $\bar{F} \cong_{w v l} G$ [10]. The following theorems from [10], [22] state a few known properties of sum graphs.

Theorem 2.1. [10] If $S \subseteq[r, 2 r]$ for some $r \in \mathbb{N}$, then $G^{+}(S)$ is totally disconnected.

Theorem 2.2. [10] Let $S=[r+l, r+n]$ with $r \in \mathbb{N}_{0}, n \geq 3$.

(a) For $n \leq r+2, G^{+}(S)$ is totally disconnected.

(b) For $n \geq r+3, G^{+}(S) \cong G_{n-r} \cup K_{r}^{c}$.

Corollary 2.3. [10] If $0 \leq r \leq n-3$ and $S=[r+l, r+n]$, then $G_{n-r} \subseteq_{w v l} G^{+}(S)$. In particular, $G_{n-2} \subseteq_{w v l} G^{+}([2, n]) \subseteq_{w v l} G_{n-1}$.

Theorem 2.4. [10] Let $k$ and $n$ be such that $2 \leq 2 k \leq n$. If $k$ pairs of supplementary vertices are removed from

(i) Harary graph $G_{n}$, then the result is isomorphic to $G_{n-2 k}$ without the vertex labels and

(ii) the graph $G_{n}^{c}$, then the result is isomorphic to $G_{n-2 k}^{c}$ without the vertex labels. 
Theorem 2.5. [13] For $r \geq 1$ and $n \geq r+2$,

(i) $G_{r} \cup G^{+}([r+1, n]) \leq G_{n} \leq G_{1}+G^{+}([2, n])$ and

(ii) $G_{1} \cup\left(G^{+}([2, n])\right)^{c} \leq G_{n}^{c} \leq G_{r}^{c}+G^{+}([r+1, n])^{c}$.

Theorem 2.6. [13] Let $n$ and $r$ be positive integers.

(a) For $n \geq 4$ and $n \geq r \geq 2$, the following pairs of labeled graphs

(i) $G_{n}$ and $G_{r}+G^{+}([r+1, n])$ and

(ii) $G_{n}^{c}$ and $G_{r}^{c} \cup\left(G^{+}([r+1, n])\right)^{c}$ are non-comparable.

(b) For $n \geq 5$, the following pairs of graphs

(i) $G_{n}$ and $G_{2}+G^{+}([3, n])$ and

(ii) $G_{n}^{c}$ and $G_{2}^{c} \cup\left(G^{+}([3, n])\right)^{c}$ without the vertex labels are non-comparable.

Theorem 2.7. [10] For $n \geq 2, G_{n} \cong_{w v l} G_{n}^{c}-\left\{(1, n),(2, n-1), \ldots,\left(\left\lfloor\frac{n}{2}\right\rfloor,\left\lceil\frac{n}{2}\right\rceil+1\right)\right\}$.

Theorem 2.8. [10] For all $n \in \mathbb{N}, G_{0, n} \cong{ }_{w v l} G_{n+1}^{c}$.

Theorem 2.9. [10] For all $m, n \in \mathbb{N}_{0}, G_{-m, n} \cup G_{0, m-1} \cup G_{0, n-1} \cong_{w v l} K_{m+n+1}$.

We have $\left|E\left(G_{n}\right)\right|=\frac{1}{2}\left(n C_{2}-\left\lfloor\frac{n}{2}\right\rfloor\right),\left|E\left(G_{n}^{c}\right)\right|=\frac{1}{2}\left(n C_{2}+\left\lfloor\frac{n}{2}\right\rfloor\right), G_{n}$ is a disconnected graph with at least one isolated vertex and $G_{n}^{c}$ is a connected graph where $\lfloor x\rfloor$ denotes the floor of $x$. In particular, we have $\left|E\left(G_{2 n}\right)\right|=n^{2}-n=\left|E\left(G_{2 n-1}^{c}\right)\right|$ and $\left|E\left(G_{2 n+1}\right)\right|=n^{2}=\left|E\left(G_{2 n}^{c}\right)\right|, n \in \mathbb{N}$.

Pythagoreans knew that squared numbers are sums of sequences of odd numbers (That is $1=1,4=1+3,9=1+3+5,16=1+3+5+7, \ldots$ ) [1] and similar to this, we obtain a relation among number of edges of $G_{2 n+1}$ and the maximal degree vertices of $G_{2 n+1}, G_{2 n-1}, \ldots, G_{3}$. Using Theorem 2.4, the underlying graphs of $G_{2 n+1}-\left\{u_{1}, u_{2 n+1}\right\}$ and $G_{2 n-1}$ are isomorphic and in $G_{2 n+1}$, vertex $u_{2 n+1}$ is nonadjacent to all other vertices and vertex $u_{1}$ is adjacent to all other vertices, except $u_{2 n+1}$ where $u_{j}$ is the vertex of $G_{2 n+1}$ with sum label $j, 1 \leq j \leq 2 n+1$. This implies, the underlying graphs of $\left\{u_{2 n+1}\right\} \cup\left(\left\{u_{2 n}\right\}+G_{2 n-1}\right)$ and $G_{2 n+1}$ are isomorphic where $u_{j}$ is the vertex of $G_{2 n+1}$ with sum label $j, 1 \leq j \leq 2 n+1$ and $n \in \mathbb{N}$. Now, using the algorithm, $\left\{u_{2 i+1}\right\} \cup\left(\left\{u_{2 i}\right\}+G_{2 i-1}\right) \cong G_{2 i+1}$ and applying the results, $\left|E\left(G_{2 i+1}\right)\right|=i^{2}$ and $\left|E\left(G_{2 i+1}\right)\right|=(2 i-1)+\left|E\left(G_{2 i-1}\right)\right|=d_{G_{2 i+1}}\left(u_{1}\right)+\left|E\left(G_{2 i-1}\right)\right|$ for $i=n, n-1, \ldots, 3$, successively, we obtain $n^{2}=\left|E\left(G_{2 n+1}\right)\right|=(2 n-1)+$ $\left|E\left(G_{2 n-1}\right)\right|=(2 n-1)+(2 n-3)+\left|E\left(G_{2 n-3}\right)\right|=\ldots=(2 n-1)+(2 n-3)+\ldots+$ $3+\left|E\left(G_{3}\right)\right|=(2 n-1)+(2 n-3)+\ldots+1=d_{G_{2 n+1}}\left(u_{1}\right)+d_{G_{2 n-1}}\left(u_{1}\right)+\ldots+d_{G_{3}}\left(u_{1}\right)$. 


\section{Decomposition of $K_{n}$ into Sum and Integral Sum Graphs}

In this section we consider decomposition of complete graphs into underlying graphs of sum and integral sum graphs. The first result follows from Theorem 2.7.

Theorem 3.1. For $3 \leq n$, the underlying graphs of $K_{n}, 2\left(G_{n}^{+}\right) \cup\left\lfloor\frac{n}{2}\right\rfloor . P_{2}$ and $G_{n}^{c} \cup\left(G_{n}^{c}-\left\{(1, n),(2, n-1), \ldots,\left(\left\lfloor\frac{n}{2}\right\rfloor, n+1-\left\lfloor\frac{n}{2}\right\rfloor\right)\right\}\right)$ are isomorphic.

Proof. Using Theorem 2.7, the underlying graphs of $G_{n}$ and $G_{n}^{c}-\{(1, n),(2, n-$ $\left.1), \ldots,\left(\left\lfloor\frac{n}{2}\right\rfloor, n+1-\left\lfloor\frac{n}{2}\right\rfloor\right)\right\}$ are isomorphic. This implies, the underlying graphs of $K_{n}=G_{n} \cup G_{n}^{c}, 2 . G_{n} \cup\left\{(1, n),(2, n-1), \ldots,\left(\left\lfloor\frac{n}{2}\right\rfloor, n+1-\left\lfloor\frac{n}{2}\right\rfloor\right)\right\}, 2\left(G_{n}^{+}\right) \cup\left\lfloor\frac{n}{2}\right\rfloor . P_{2}$ and $G_{n}^{c} \cup\left(G_{n}^{c}-\left\{(1, n),(2, n-1), \ldots,\left(\left\lfloor\frac{n}{2}\right\rfloor, n+1-\left\lfloor\frac{n}{2}\right\rfloor\right)\right\}\right)$ are isomorphic. Hence the result.

Theorem 3.2. The underlying graphs of $G_{0, n}$ and $G_{n+1}^{c}$ are isomorphic, $n \in \mathbb{N}$.

Proof. Using the definition of anti-sum labeling, we obtain, $G_{n+1}^{c}=\left(\left(\left(G_{n}^{c} \cup\right.\right.\right.$ $\left.\left.K_{1}(n+1)\right)-\left\{(1, n),(2, n-1), \ldots,\left(\left\lfloor\frac{n}{2}\right\rfloor, n+1-\left\lfloor\frac{n}{2}\right\rfloor\right)\right\}\right) \cup\{(1, n+1),(2, n+$ $1), \ldots,(n, n+1)\}) \cong\left(G_{n}^{c}-\left\{(1, n),(2, n-1), \ldots,\left(\left\lfloor\frac{n}{2}\right\rfloor, n+1-\left\lfloor\frac{n}{2}\right\rfloor\right)\right\}\right) \cup K_{1}(n+$ 1) $\cup\{(1, n+1),(2, n+1), \ldots,(n, n+1)\}$ which is isomorphic to the underlying graph of $G_{n} \cup K_{1}(n+1) \cup\{(1, n+1),(2, n+1), \ldots,(n, n+1)\}=G_{n}+K_{1}(n+1)$ where $K_{1}(n+1)$ represents a vertex with vertex label $n+1$ in the graph $G_{n}^{c} \cup K_{1}(n+1)$. Thus, the underlying graphs of $G_{n+1}^{c}, G_{n}+K_{1}(n+1)$ and $G_{0, n}$ are isomorphic since the underlying graphs of $G_{n}+K_{1}(n+1)$ and $G_{0, n}$ are isomorphic. Hence the result.

Theorem 3.3. For $m, n \in \mathbb{N}_{0}$, the underlying graphs of $K_{m+n+1}$ and $G_{-m, n} \cup$ $G_{0, m-1} \cup G_{0, n-1}$ are isomorphic.

Proof. For $m, n \in \mathbb{N}_{0}, G_{-m, n}=K_{1}+\left(\left(-G_{m}\right)+G_{n}\right)$ and hence, $G_{-m, n}^{c}=$ $K_{1}(0) \cup\left(-G_{m}\right)+G_{n}{ }^{c}=K_{1}(0) \cup\left(-G_{m}^{c}\right) \cup G_{n}^{c}$ which is isomorphic to the underlying graph of $K_{1}(0) \cup G_{0, m-1} \cup G_{0, n-1}$, using Theorem 3.2. Hence the result.

Corollary 3.4. For $2 \leq n$, the underlying graphs of $K_{2 n}, G_{-(n-1), n} \cup G_{0, n-2} \cup G_{0, n-1}$ and 2. $G_{n} \cup n . P_{2}$ are isomorphic and that of $K_{2 n+1}$ and $G_{-n, n} \cup 2 . G_{0, n-1}$ are isomorphic.

Proof. For $2 \leq n$, using Theorem 3.3, we obtain that the underlying graphs of $K_{2 n}$ and $G_{-(n-1), n} \cup G_{0, n-2} \cup G_{0, n-1}$ are isomorphic and the underlying graphs of $K_{2 n+1}$ and $G_{-n, n} \cup 2 . G_{0, n-1}$ are isomorphic and using Theorem 3.1, we obtain that the underlying graphs of $K_{2 n}$ and $G_{-(n-1), n} \cup G_{0, n-2} \cup G_{0, n-1} \cong 2 . G_{n} \cup n . P_{2}$ are isomorphic. Hence the result.

\section{A Few Properties of Integral Sum graphs $G_{0, n}$}

In this section we obtain a few structural properties of integral sum graphs $G_{0, n}, n \in \mathbb{N}$. 
Theorem 4.1. For $n \geq 3$, the underlying graphs of $G_{0, n}-\{0, n\}$ and $G_{0, n-2}$ are isomorphic.

Proof. Let $V\left(G_{0, n-2}\right)=\left\{u_{0}, u_{1}, \ldots, u_{n-2}\right\}$ and $V\left(G_{0, n}\right)=\left\{v_{0}, v_{1}, \ldots, v_{n}\right\}$ where $i$ and $j$ are integral sum labels of $u_{i}$ and $v_{j}$, respectively, $0 \leq i \leq n-2$ and $0 \leq j \leq n$. Define mapping $f: V\left(G_{0, n-2}\right) \rightarrow V\left(G_{0, n}\right)$ such that $f\left(u_{i}\right)=v_{i+1}$ for $i=0,1, \ldots, n-2$. Now, $u_{i}$ and $u_{j}$ are adjacent in $G_{0, n-2}$ if and only if $i \neq j, 0 \leq i, j \leq n-2$ and $0+1=1 \leq i+j \leq n-2$ if and only if $i+1 \neq j+1,1 \leq$ $i+1, j+1 \leq n-1$ and $1+2=3 \leq(i+1)+(j+1) \leq n=(n-1)+1$ if and only if $v_{i+1}$ and $v_{j+1}$ are adjacent in $G_{0, n}-\{0, n\}$ if and only if $f\left(u_{i}\right)$ and $f\left(u_{j}\right)$ are adjacent in $G_{0, n}-\{0, n\}$. This implies the mapping $f$ is bijective, preserves adjacency and $f\left(G_{0, n}-\{0, n\}\right)=G_{0, n-2}$.

Theorem 4.2. For $n \geq 5$, the underlying graphs of $G_{0, n}-(\{0, n, n-1, n-2\} \cup$ $[n] \cup[n-1])$ and $G_{0, n-4}$ are isomorphic.

PROOF. Using the definition of integral sum labeling we obtain isomorphic graphs of the underlying graphs of $G_{0, n}-(\{n, n-1\} \cup[n] \cup[n-1])$ and $G_{0, n-2}$ where $[k]$ in $G^{+}(S)$ denotes the set of all edges of $G^{+}(S)$ whose edge sum value is $k, k \in S$ [16]. Using Theorem 4.1, the underlying graphs of $G_{0, n-2}-\{0, n-2\}$ and $G_{0, n-4}$ are isomorphic. Hence the result.

Generalizing the above Theorem, we obtain the following result.

Theorem 4.3. For $n \geq 3$, the underlying graphs of $G_{0, n}-\{0, n\}$ and $G_{0, n-2}$ are isomorphic and for $n \geq 2 r+3$ and $r \in \mathbb{N}$, the underlying graphs of $G_{0, n}-(\{0, n, n-$ $1, n-2, \ldots, n-2 r+1, n-2 r\} \cup([n] \cup[n-1] \cup \ldots \cup[n-2 r+1]))$ and $G_{0, n-2 r-2}$ are isomorphic.

Theorem 4.4. For $5 \leq n$, the underlying graphs of $G_{0, n}-\{0,1, n-1, n\}$ and $G_{0, n-4}$ are isomorphic where $u_{j}$ is the vertex of $G_{0, n}$ with integral sum label $j, j=$ $0,1, \ldots, n$.

Proof. Using Theorem 3.2, the underlying graphs of $G_{0, n}$ and $G_{n+1}^{c}$ are isomorphic and from the structure of these graphs (See graphs $G_{8}^{c}$ in Figures 6 and $G_{0,7}$ in Figure 7.), vertex with integral sum label $j$ in $G_{0, n}$ and vertex with anti-integral sum label $n-j+1$ in $G_{n+1}^{c}$ are of same degree and thereby the underlying graphs of $G_{0, n}-\{0,1, n-1, n\}$ and $G_{n+1}^{c}-\{n+1, n, 2,1\}$ are isomorphic, $0 \leq j \leq n$. Using Theorem 2.4, the underlying graphs of $G_{n+1}^{c}-\{1,2, n, n+1\}$ and $G_{n-3}^{c}$ are isomorphic and using Theorem 3.2, the underlying graphs of $G_{n-3}^{c}$ and $G_{0, n-4}$ are isomorphic. This implies, the underlying graphs of $G_{0, n}-\{0,1, n-1, n\}$ and $G_{0, n-4}$ are isomorphic. Hence the result.

Theorem 4.5. For $n \geq 3$, the following pairs of underlying graphs of

(i) $\left(G_{0, n-1}\right)^{c}$ and $G_{0, n-2} \cup K_{1}(n-1)$ and 


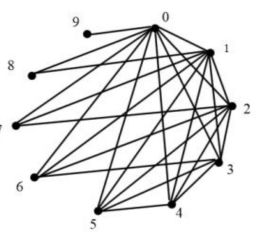

$\mathrm{G}_{0,9}$

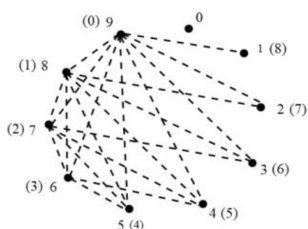

$\mathbf{G}_{0,8}$

Fig. 10.

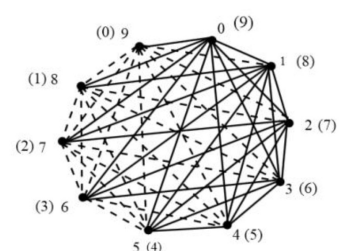

Fig. 11: $K_{10} \cong G_{0,9} \cup\left(G_{0,8} \cup K_{1}(9)\right)$.

(ii) $K_{n}$ and $G_{0, n-1} \cup\left(G_{0, n-2} \cup K_{1}(n-1)\right)$ are isomorphic where $K_{1}(n-1)$ is an isolated vertex with label $n-1$.

ProOF. We have the underlying graphs of $\left(G_{0, n-1}\right)^{c}=$ the underlying graphs of $\left(K_{1}(0)+G_{n-1}\right)^{c}=$ the underlying graphs of $K_{1}(0) \cup G_{n-1}^{c}$ whose underlying graph is isomorphic to the underlying graph of $K_{1}(0) \cup G_{0, n-2}$ which is isomorphic to the underlying graph of $K_{1}(n-1) \cup G_{0, n-2}$, using Theorem 3.2 where $K_{1}(0)$ and $K_{1}(n-1)$ are isolated vertices in the underlying graphs of $K_{1}(0) \cup G_{0, n-2}$ and $K_{1}(n-1) \cup G_{0, n-2}$, respectively. This implies the underlying graphs of $\left(G_{0, n-2} \cup\right.$ $\left.K_{1}(n-1)\right)^{c}$ and $G_{0, n-1}$ are isomorphic. This implies the underlying graphs of $K_{n}$ and $G_{0, n-1} \cup\left(G_{0, n-2} \cup K_{1}(n-1)\right)$ are isomorphic where $K_{1}(n-1)$ is an isolated vertex with label $n-1$. Hence the result. See Figures 10 and 11 .

\section{Two New Families of Integral Sum Graphs}

In [18] it is proved that fan graph $F_{n}=P_{n}+K_{1}$ is an integral sum graph, $n \geq 2$. In this section we obtain two new families of integral sum graphs, namely, triangular book with a book mark and fan with a handle.

Theorem 5.1. For $n \in \mathbb{N}$,

(i) $T B_{n}\left(u_{0}, v_{0}\right)\left(u_{0}, w_{0}\right)$ and

(ii) $F_{n}^{*}$ are integral sum graphs.

ProOF.

(i) $T B_{n}\left(u_{0}, v_{0}\right)\left(u_{0}, w_{0}\right)$ is of order $n+3$, size $2 n+2$ and $\left(u_{0}, w_{0}\right)$ is the pendant edge terminating at $u_{0}$. Let $V\left(T B_{n}\left(u_{0}, v_{0}\right)\left(u_{0}, w_{0}\right)\right)=\left\{w_{0}, u_{0}, v_{0}, v_{1}, \ldots\right.$, $\left.v_{n}\right\}$. Define mapping $f: V\left(T B_{n}\left(u_{0}, v_{0}\right)\left(u_{0}, w_{0}\right)\right) \rightarrow \mathbb{N}_{0}$ such that $f\left(u_{0}\right)=0$, $f\left(v_{0}\right)=2 m, f\left(v_{i}\right)=2 m i+1$ for $i=1,2, \cdots, n$ and $f\left(w_{0}\right)=2 m(n+$ $1)+1, m \in \mathbb{N}$. Consider the integral sum graph $G^{+}(S)$ where $S=$ $\{0,2 m, 2 m+1,4 m+1,6 m+1, \ldots, 2 m n+1,2 m(n+1)+1 / m \in \mathbb{N}\}=$ $f\left(V\left(T B_{n}\left(u_{0}, v_{0}\right)\left(u_{0}, w_{0}\right)\right)\right)$. Our aim is to prove that $G^{+}(S)=T B_{n}\left(u_{0}, v_{0}\right)\left(u_{0}, w_{0}\right)$. $f\left(u_{0}\right)=0$ implies, $f\left(u_{0}\right)+f\left(v_{i}\right)=f\left(v_{i}\right)$ and $f\left(u_{0}\right)+f\left(w_{0}\right)=f\left(w_{0}\right)$ for $i=0,1,2, \ldots, n$. This implies, $u_{0}$ is adjacent to $w_{0}, v_{0}$ and $v_{i}$ for 
$i=1,2, \ldots, n$. For $i=1,2, \ldots, n-1, f\left(v_{0}\right)+f\left(v_{i}\right)=f\left(v_{i+1}\right), f\left(v_{0}\right)+f\left(v_{n}\right)$ $=f\left(w_{0}\right), f\left(v_{0}\right)+f\left(u_{0}\right)=f\left(v_{0}\right), f\left(v_{0}\right)+f\left(w_{0}\right) \neq f\left(u_{0}\right), f\left(v_{0}\right), f\left(w_{0}\right), f\left(v_{j}\right)$ for $j=1,2, \ldots, n$. This implies $v_{0}$ is adjacent to $u_{0}$ and $v_{i}$ and nonadjacent to $w_{0}$ for $i=1,2, \ldots, n$. Also $f\left(w_{0}\right)+f\left(u_{0}\right)=f\left(w_{0}\right)$ and $f\left(w_{0}\right)+f\left(v_{j}\right) \neq f\left(w_{0}\right), f\left(u_{0}\right), f\left(v_{j}\right)$ for $j=0,1, \ldots, n$. This implies, $w_{0}$ is a pendant vertex adjacent only to $u_{0}$.

For $i, j=0,1,2, \ldots, n, f\left(v_{i}\right)+f\left(w_{0}\right) \neq f\left(u_{0}\right), f\left(v_{j}\right)$. Also for $1 \leq$ $i, j, k \leq n, f\left(v_{i}\right)+f\left(v_{j}\right) \neq f\left(v_{k}\right)$ since $f\left(v_{i}\right)+f\left(v_{j}\right)$ is an even number and $f\left(v_{k}\right)$ is an odd number. This implies, $v_{i}$ and $v_{j}$ are non-adjacent in $T B_{n}\left(u_{0}, v_{0}\right)\left(u_{0}, w_{0}\right)$ when $i \neq j$ and $1 \leq i, j \leq n$. Thus $v_{j}$ is adjacent only to $u_{0}$ and $v_{0}$ for $j=1,2, \ldots, n$. From all the above conditions integral sum graph $G^{+}(S)=T B_{n}\left(u_{0}, v_{0}\right)\left(u_{0}, w_{0}\right)$ where $S=\{0,2 m, 2 m+1,4 m+$ $1, \ldots, 2 m n+1,2 m(n+1)+1 / m \in \mathbb{N}\}$. Integral sum labeling of $T B_{7}^{*}$ is shown in Figure 12.

(ii) Fan graph $F_{n}=P_{n}+K_{1}$ and $F_{n}^{*}$ is of order $n+2$ and size $2 n$ where $P_{n}$ is a path on $n$ vertices. Let $V\left(F_{n}^{*}\right)=\left\{u_{0}, v_{0}, v_{1}, \ldots, v_{n}\right\}$ where $u_{0}$ is the pendant vertex, $v_{0}$ is the apex vertex and $d\left(v_{0}\right)=n+1=\Delta\left(F_{n}^{*}\right)$. Define mapping $f: V\left(F_{n}^{*}\right) \rightarrow \mathbb{N}$ such that $f\left(v_{0}\right)=0, f\left(v_{1}\right)=p_{m}$, the $m^{\text {th }}$ Fibonacci number, $m \geq 2, f\left(v_{i}\right)=p_{m+i-1}$ for $i=2, \ldots, n$ and $f\left(u_{0}\right)=p_{m+n}$. Here, $f\left(v_{0}\right)=0<f\left(v_{1}\right)=p_{m}<f\left(v_{2}\right)=p_{m+1}<\ldots<f\left(v_{n}\right)=p_{m+n-1}<$ $f\left(u_{0}\right)=p_{m+n}$ and for $i-j \neq 1$ and $1 \leq i, j, k \leq n, f\left(v_{i}\right)+f\left(v_{j}\right) \neq f\left(v_{k}\right)$. Also $f\left(v_{i}\right)+f\left(v_{i+1}\right)=f\left(v_{i+2}\right)$ for $i=1,2, \ldots, n-2$ and $f\left(v_{n-1}\right)+f\left(v_{n}\right)=$ $f\left(u_{0}\right), m \geq 2$. Hence the labeling $f$ is an integral sum labeling of graph $F_{n}^{*}$. Integral sum labeling of $F_{9}^{*}$ is shown in Figure 12 .

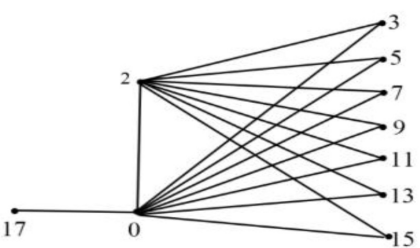

Fig. 12:

$T B_{7}^{*}$.

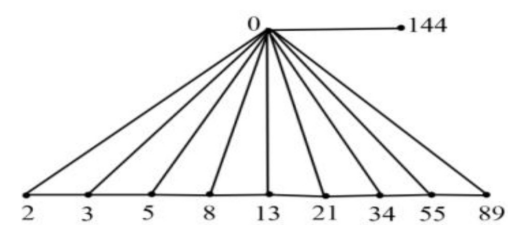

$F_{9}^{*}$.

Acknowledgement Research supported in part by DST, Government of India, under grant SR/S4/MS: 679/10 (DST-SERB) and Lerroy Wilson Foundation, Nagercoil, India (Face Book: Lerroy Wilson Foundation, India).

\section{REFERENCES}

[1] Amird Aczel, Fermatfs last theorem, Dell Publishing, NY, 1997, page-20. 
[2] Z. Chen, Harary's conjecture on integral sum graphs, Discrete Math., 160 (1990), 241-244.

[3] Douglas B. West, Introduction to graph theory, Pearson Education, 2005.

[4] M. N. Ellingham, Sum Graphs from trees, Ars Comb., 35 (1993), 335-349.

[5] J.A. Gallian, A dynamic survey of graph labeling, Electronic J. Comb., 19 (2013), \#DS6.

[6] F. Harary, Graph Theory, Addison Wesley, Reading Mass., 1969.

[7] F. Harary, Sum graphs and difference graphs, Cong. Num., 72 (1990), 101-108.

[8] F. Harary, Sum graphs over all integers, Discrete Math., 124 (1994), 99-105.

[9] T. Nicholas, S. Somasundaram and V. Vilfred, Some results on sum graphs, J. Comb. Inf. \& System Sci., 26 (2001), 135.142.

[10] V. Vilfred, L.W. Beineke and A. Suryakala, More properties of sum graphs, Graph Theory Notes of New York, MAA, 66 (2014), 10-15.

[11] V. Vilfred, R. Kala and A. Suryakala, $(a, d)$-Ascending subgraph decomposition of graphs $K_{n}$ and $G_{0, n}$, Proc. Int. Conf. on App. Math. and Theoretical Comp. Sci., St. Xavierfs College of Engg., Nagercoil, TN, India (2013), 296-303.

[12] V. Vilfred, R. Kala and A. Suryakala, Number of Triangles in Integral Sum Graphs $G_{m, n}$, IJ of Algorithms, Computing and Mathematics, 4 (2011), 16-24.

[13] V. Vilfred and L. Mary Florida, Anti-integral sum graphs and decomposition of $G_{n}, G_{n}^{c}$ and $K_{n}$, Proc. Int. Conf. on App. Math. and Theoretical Comp. Sci., St. Xavierfs College of Engg., Nagercoil, TN, India (2013), 129-133.

[14] V. Vilfred and L. Mary Florida, Integral sum graphs and maximal integral sum graphs, Graph Theory Notes of New York, MAA, 63 (2012), 28-36.

[15] V. Vilfred and L. Mary Florida, Integral sum graphs $H_{X, Y}^{R, T}$, edge sum class and edge sum color number, Proc. Int. Conf. on Math. in Engg. and Business Management, Stella Maris College, Chennai, India (2012), 88-94.

[16] V. Vilfred and L. Mary Florida, New families of integral sum graphs - edge sum class and chromatic integral sum number, Proc. Int. Conf. on App. Math. and Theoretical Comp. Sci., St. Xavierfs Catholic College of Engg., Nagercoil, TN, India (2013), 177-182.

[17] V. Vilfred and L. Mary Florida, Sum number and exclusiveness of graphs $C_{4}, L_{n}$ and $P_{3} \square P_{3}$ Proc. Inter. Conf. on Math. in Engg. and Business Management, Stella Maris College, Chennai, India (2012), 13-15.

[18] V. Vilfred and T. Nicholas, Amalgamation of integral sum graphs, fan and Dutch $M-$ Windmill are integral sum graphs, Graph Theory Notes of New York, MAA, 58 (2010), 51-54.

[19] V. Vilfred and T. Nicholas, Banana trees and union of stars are integral sum graphs, Ars Comb., 102 (2011), 79-85.

[20] V. Vilfred and T. Nicholas, The integral sum graph $G_{\Delta n}$, Graph Theory Notes of New York, $M A A, 57$ (2009), 43-47.

[21] V. Vilfred and K. Rubin Mary, Number of Cycles of Length Four in the Integral Sum Graphs $G_{m, n}$, Proc. Inter. Conf. on App. Math. and Theoretical Comp. Sci., St. Xavierfs College of Engg., Nagercoil, TN, India (2013), 134-141.

[22] V. Vilfred and A. Suryakala, More properties of sum graphs, Proc. Int. Conf. on App. Math. and Theoretical Comp. Sci., St. Xavierfs College of Engg., Nagercoil, TN, India (2013), 142145 .

[23] V. Vilfred, A. Suryakala and K. Rubin Mary, More on integral sum graphs, Proc. Int. Conf. on App. Math. and Theoretical Comp. Sci., St. Xavierfs College of Engg., Nagercoil, TN, India (2013), 173-176. 